\title{
VOCO
}

\section{Die Vorteile von Composite und Glasionomer in einem Produkt}

Ionolux ist ein lichthärtendes Glasionomer-Füllungsmaterial in den Farben A1, A2 und A3, das für die Anwendung bei verschiedenen Indikationen in der konservierenden und präprothetischen Zahnheilkunde entwickelt wurde. Es lässt sich hervorragend in die Kavität einbringen, blockt Unterschnitte aus, nivelliert Unebenheiten und führt dabei nicht zu Änderungen der natürlichen Farbe der umgebenden Zahnhartsubstanz. Das Produkt ist außerdem sehr gut für die Sandwich-Technik geeignet, da es mit allen konventionellen lichthärtenden Füllungsmaterialien kompatibel ist. Mit seinen optimalen Material- und Handling-Eigenschaften empfiehlt es sich für den Einsatz gerade auch dort, wo Patienten eine besondere Herausforderung für den Zahnarzt darstellen, etwa in der Kinder- oder der Alterszahn- heilkunde sowie in der Schmerz- und Notfall-Therapie. Ionolux bietet sich zudem für präprothetische Versorgungen an, bei denen ausgedehnte Zahnhartsubstanzdefekte behoben werden müssen, um anschließend Aufbauten präparieren und permanente Restaurationen vornehmen zu können. Es lässt sich nach lediglich relativer Trockenlegung schnell applizieren. Das Material ist sofort nach Einbringen in die Kavität stopf- und modellierbar ohne am Instrument zu kleben und lässt sich ausgezeichnet an die Kavitätenwände adaptieren. Aufgrund seiner spezifischen Materialzusammensetzung ist lonolux einfach zu polieren und biokompatibel. Durch seine Fluoridabgabe trägt es dazu bei, das Risiko von Sekundärkaries zu mindern. Zusätzlich verfügt es über eine hohe Druckhärte sowie eine

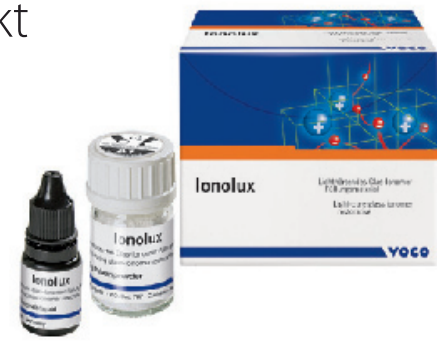

geringe Wasserlöslichkeit und sorgt damit für stabile, dauerhafte Restaurationen. Neben der bereits eingeführten praktischen AC-Variante gibt es nun auch die besonders wirtschaftliche Handmischvariante.

入 Tel. 04721 - 719-0

www.voco.de

medentex

\section{Bei jedem Behälter spart die Praxis bis zu 50 Euro!}

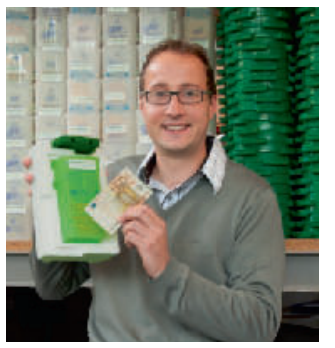

Frohe Kunde für Zahnarztpraxen, die Metasys Systeme aus Österreich zur Amalgamabscheidung verwenden. In umfangreichen Testreihen konnte nun nachgewiesen werden, dass selbst 12 Jahre alte Auffangbehälter ihre nachhaltige Qualität beweisen. Die hohe Haltbarkeit der Behälter ist nicht nur für die Betriebssicherheit der Systeme von Bedeutung. Die solide Qualität aus Tirol stellt sicher, dass auch diese Behälter über viele Jahre im Mehrweg-Kreislauf eingesetzt werden können und die EU-Anforderungen für Gefahrguttransporte (ADR) in diesen Mengen problemlos erfüllen.

Durchgeführt wurden die Qualitätstests und wissenschaftlichen Expertisen von der medentex $\mathrm{GmbH}$ in Bielefeld. Der zertifizierte Umweltbetrieb gilt als der führende Spezialist für die Entsorgung von Amalgamschlämmen in Europa. Inzwischen nutzt hierzulande jede zweite Zahnarztpraxis den medentex Umweltservice.
„Rund 50 Euro kann die Zahnarztpraxis pro Behälter sparen, wenn man sich für das Recycling im Mehrwegsystem entscheidet. Wieder zeigt sich, dass Umweltschutz auch unter wirtschaftlichen Aspekten eine echte Chance ist", kommentiert Christian Finke, Unternehmensleitung medentex, die Testergebnisse.

入 Tel. 05205 - 7516-0 info@medentex.de www.medentex.de

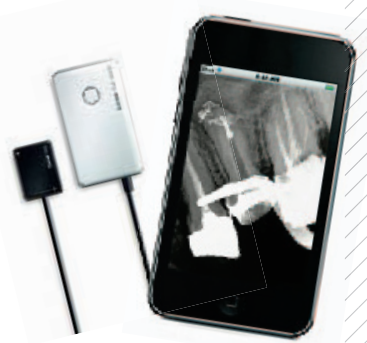

men Tomographie 3D System habe man Anfang 2009 das digitale Röntgen-Portfolio auch um ein Gerät für die Erfassung und Darstellung mittlerer und großer Volumen erweitert, das eine weitere "Gewinnergeschichte" schreibt.

ス Tel. 0711 - 20707-306 europedental@cshdental.com www.kodakdental.com 